ESAIM: PROCEEDINGS AND SURVEYS, September 2014, Vol. 45, p. 18-31

J.-S. Dhersin, Editor

\title{
HOMOGENIZATION THEORY AND MULTISCALE NUMERICAL APPROACHES FOR DISORDERED MEDIA: SOME RECENT CONTRIBUTIONS ${ }^{*, * *}$
}

\author{
Claude Le Bris ${ }^{1}$
}

\begin{abstract}
We overview a series of recent works related to some multiscale problems motivated by practical problems in Mechanics. The common denominator of all these works is that they address multiscale problems where the geometry of the microstructures is not periodic. Random modelling, as well as other types of nonperiodic modelling, can then be used to account for the imperfections of the medium. The theory at play is that of homogenization, in its many variants (stochastic, general deterministic, periodic). The numerical methods developed and adapted are finite element type methods. A special emphasis is laid on situations where the amount of randomness is small, or, put differently, when the disorder is limited. Then, specific, computationally efficient techniques can be designed and employed.

Résumé. Nous présentons un panorama d'une série de travaux récents sur des problèmes multiéchelles motivés par la science des matériaux. Le dénominateur commun de ces travaux est qu'ils traitent tous de problèmes où la géométrie des microstructures n'est pas périodique. La modélisation aléatoire, ainsi que d'autres types de modélisations non periodiques, sont alors utilisées pour rendre compte des imperfections du matériau. Le cadre théorique est celui de l'homogénéisation dans ses nombreuses variantes (stochastique, déterministique générale, périodique). Les méthodes numériques développées et adaptées sont des méthodes de type éléments finis. Une attention toute particulière est portée sur les problèmes où la "quantité de hasard" est petite ou, autrement dit, le désordre est limité. Des techniques spécifiques, efficaces pour le calcul, peuvent alors être définies et utilisées.
\end{abstract}

\section{INTRODUCTION}

We overview a series of recent works related to some multiscale problems motivated by practical problems in Mechanics. The common denominator of all these works is that they address multiscale problems where the geometry of the microstructures is not periodic. For simplicity, we argue on a linear elliptic scalar equation.

We begin by recalling in Section 1 the basics of homogenization theory, both in the deterministic (periodic) context and the random context, which are useful for our exposition. Then, in Section 2, we summarize a series of works devoted to what we call "weakly" random settings, that is, settings where the random disorder is small

\footnotetext{
* The work of the author is partially supported by EOARD under Grant (No. FA8655-13-1-3061) and by ONR under Grant (No. N00014-12-1-0383).

** The author would like to thank his many friends and collaborators on the issues presented here, in particular X. Blanc (Paris 7), P.-L. Lions (Collège de France), F. Legoll, W. Minvielle (Ecole des Ponts and Inria), A. Lozinski (Besançon). The material covered in this survey article has been presented as a plenary lecture at SMAI 2013. The author is grateful to the organizing and scientific committees for providing this opportunity to present his work.

${ }^{1}$ Ecole des Ponts and INRIA, 6 \& 8 avenue Blaise Pascal, 77455 Marne-la-Vallée Cedex 2, France. lebris@cermics.enpc.fr
}

(c) EDP Sciences, SMAI 2014 
and does not significantly perturb an original ordered structure (here taken as periodic). Section 3 addresses the somewhat opposite case, when randomness cannot be considered small and thus variance is intrinsically large. Variance reduction techniques, adapted to the specific context of homogenization, are described and tested. Our final Section 4 presents another research direction. We use an entirely deterministic setting to model disordered structures and yet conduct an explicit homogenization process.

Before we proceed, we mention that we will assume throughout our text that the reader is reasonably familiar with homogenization theory on the one hand and finite element methods on the other hand. We refer to the classical textbooks $[5,21]$ and $[15,31]$, respectively, for those topics.

\section{BRIEF OVERVIEW OF CLASSICAL HOMOGENIZATION SETTINGS}

\subsection{Periodic homogenization}

To begin with, we recall some well known, basic ingredients of elliptic homogenization theory in the periodic setting. We consider, in a regular domain $\mathcal{D}$ in $\mathbb{R}^{d}$, the problem

$$
\left\{\begin{array}{l}
-\operatorname{div}\left[A_{\text {per }}\left(\frac{x}{\varepsilon}\right) \nabla u^{\varepsilon}\right]=f \text { in } \mathcal{D} \\
u^{\varepsilon}=0 \text { on } \partial \mathcal{D}
\end{array}\right.
$$

where the matrix $A_{\text {per }}$ is symmetric and $\mathbb{Z}^{d}$-periodic. We manipulate for simplicity symmetric matrices, but our discussion carries over to non symmetric matrices up to slight modifications.

The corrector problem associated to (1) reads, for $p$ fixed in $\mathbb{R}^{d}$,

$$
\left\{\begin{array}{l}
-\operatorname{div}\left(A_{\text {per }}(y)\left(p+\nabla w_{p}\right)\right)=0 \\
w_{p} \text { is } \mathbb{Z}^{d} \text {-periodic. }
\end{array}\right.
$$

It has a unique solution up to the addition of a constant. Then, the homogenized coefficients read

$$
\left[A_{*}\right]_{i j}=\int_{Q} e_{i}^{T} A_{p e r}(y)\left(e_{j}+\nabla w_{e_{j}}(y)\right) d y
$$

where $Q$ is the unit cube. The main result of periodic homogenization theory is that, as $\varepsilon$ goes to zero, the solution $u^{\varepsilon}$ to $(1)$ converges to $u^{*}$ solution to

$$
\left\{\begin{array}{l}
-\operatorname{div}\left[A_{*} \nabla u^{*}\right]=f \quad \text { in } \quad \mathcal{D} \\
u^{*}=0 \text { on } \partial \mathcal{D}
\end{array}\right.
$$

The convergence holds in $L^{2}(\mathcal{D})$, and weakly in $H_{0}^{1}(\mathcal{D})$. The correctors $w_{e_{i}}$ (for $e_{i}$ the canonical vectors of $\mathbb{R}^{d}$ ) may then also be used to "correct" $u^{*}$ in order to identify the behavior of $u^{\varepsilon}$ in the strong topology $H_{0}^{1}(\mathcal{D})$.

Practically, at the price of only computing $d$ periodic problems (2), the solution to problem (1) can be efficiently approached for $\varepsilon$ small.

\subsection{Stochastic homogenization}

Because this is well known and for the sake of brevity, we skip all technicalities related to the definition of the probabilistic setting, which we assume discrete stationary and ergodic (we refer e.g. to [1] for all details). We now fix $A(., \omega)$ a square matrix of size $d$, which is assumed stationary in the sense

$$
\forall k \in \mathbb{Z}^{d}, \quad A(x+k, \omega)=A\left(x, \tau_{k} \omega\right) \text { almost everywhere in } x, \text { almost surely }
$$


(where $\tau$ is an ergodic group action) and which is assumed to enjoy the classical assumptions of uniform ellipticity and boundedness. Then we consider the boundary value problem

$$
\left\{\begin{array}{l}
-\operatorname{div}\left(A\left(\frac{x}{\varepsilon}, \omega\right) \nabla u^{\varepsilon}\right)=f \text { in } \mathcal{D}, \\
u^{\varepsilon}=0 \text { on } \partial \mathcal{D} .
\end{array}\right.
$$

Standard results of stochastic homogenization [5,21] apply and allow to find the homogenized problem for problem (6). These results generalize the periodic results recalled in Section 1.1. The solution $u^{\varepsilon}$ to (6) converges to the solution to (4) where the homogenized matrix is now defined as:

$$
\left[A_{*}\right]_{i j}=\mathbb{E}\left(\int_{Q} e_{i}^{T} A(y, \cdot)\left(e_{j}+\nabla w_{e_{j}}(y, \cdot)\right) d y\right),
$$

where for any $p \in \mathbb{R}^{d}, w_{p}$ is the solution (unique up to the addition of a random constant) to

$$
\left\{\begin{array}{l}
-\operatorname{div}\left[A(y, \omega)\left(p+\nabla w_{p}(y, \omega)\right)\right]=0, \quad \text { a.s. on } \mathbb{R}^{d} \\
\nabla w_{p} \quad \text { is stationary in the sense of }(5) \\
\mathbb{E}\left(\int_{Q} \nabla w_{p}(y, \cdot) d y\right)=0
\end{array}\right.
$$

A striking difference between the stochastic setting and the periodic setting can be observed comparing (2) and (7). In the periodic case, the corrector problem is posed on a bounded domain (namely, the periodic cell $Q$ ), since the corrector $w_{p}$ is periodic. In sharp contrast, the corrector problem (7) of the random case is posed on the whole space $\mathbb{R}^{d}$, and cannot be reduced to a problem posed on a bounded domain. The fact that the random corrector problem is posed on the entire space has far reaching consequences for numerical practice. Truncations of problem (7) have to be considered, typically on large domains $Q_{N}$ and using periodic boundary conditions. The actual homogenized coefficients are only captured in the asymptotic regime. Important theoretical questions about the quality and the rate of the convergence in terms of the truncation size arise (see, in particular, the pioneering works by A. Bourgeat and A. Piatnitski [13,14] and a recent series of works by A. Gloria and F. Otto [19]).

We conclude this section by mentioning that all the theoretical and numerical developments we will expose in the subsequent sections are explained in a context where one approximates numerically the corrector solution to (2) (or, respectively (7)) and next the homogenized coefficient matrix $A^{*}$ in (4). Our modeling strategy and our discussion may also be considered in the context of numerical approximation techniques in the vein of Multiscale Finite Element Methods (MsFEM), where homogenization theory "only" serves as a guideline for the construction of the approach and also as an ingredient for the numerical analysis. The work [22] presents an example of possible applications in this context. Likewise, the works $[25,26]$ show examples of difficulties created by a disordered microstructure when applying techniques such as MsFEM, and how these difficulties may be circumvented. 


\section{SOME VARIANTS OF STOCHASTIC HOMOGENIZATION AND THEIR APPLICATION TO "WEAKLY RANDOM" SETTINGS}

We now review some variants of the previous settings (see also [23]). These variants essentially encode imperfections with respect to the perfect (here periodic) context.

\subsection{Random deformations of the periodic setting}

The following specific stochastic setting has been introduced and studied in [10]. It is motivated by the consideration of random materials that have some relation to the periodic setting, taken as a reference configuration. The actual configuration is seen via a random mapping from the reference configuration.

We fix some $\mathbb{Z}^{d}$-periodic, square matrix $A_{\text {per }}$ of size $d$, assumed to satisfy the "usual" properties and we consider

$$
\left\{\begin{array}{l}
-\operatorname{div}\left(A_{\text {per }}\left(\Phi^{-1}\left(\frac{x}{\varepsilon}, \omega\right)\right) \nabla u^{\varepsilon}\right)=f \text { in } \mathcal{D} \\
u^{\varepsilon}=0 \text { on } \partial \mathcal{D}
\end{array}\right.
$$

where the function $\Phi(\cdot, \omega)$ is assumed to be a diffeomorphism from $\mathbb{R}^{d}$ to $\mathbb{R}^{d}$ for almost every $\omega$. The diffeomorphism, called a random stationary diffeomorphism, is assumed to additionally satisfy

$$
\begin{aligned}
& \inf _{\omega \in \Omega, x \in \mathbb{R}^{d}}[\operatorname{det}(\nabla \Phi(x, \omega))]=\nu>0, \\
& \sup _{\omega \in \Omega, x \in \mathbb{R}^{d}}(|\nabla \Phi(x, \omega)|)=M<\infty, \\
& \nabla \Phi(x, \omega) \quad \text { is stationary in the sense of }(5) .
\end{aligned}
$$

Then homogenization holds for the above problem (the details are made precise in [10]). The homogenized problem again reads as in (4) with the homogenized matrix given by:

$$
\left[A_{*}\right]_{i j}=\operatorname{det}\left(\mathbb{E}\left(\int_{Q} \nabla \Phi(z, \cdot) d z\right)\right)^{-1} \mathbb{E}\left(\int_{\Phi(Q, \cdot)} e_{i}^{T} A_{p e r}\left(\Phi^{-1}(y, \cdot)\right)\left(e_{j}+\nabla w_{e_{j}}(y, \cdot)\right) d y\right)
$$

where for any $p \in \mathbb{R}^{d}, w_{p}$ is the solution (unique up to the addition of a random constant and belonging to the appropriate functional space) to

$$
\left\{\begin{array}{l}
-\operatorname{div}\left[A_{p e r}\left(\Phi^{-1}(y, \omega)\right)\left(p+\nabla w_{p}\right)\right]=0, \\
w_{p}(y, \omega)=\tilde{w}_{p}\left(\Phi^{-1}(y, \omega), \omega\right), \quad \nabla \tilde{w}_{p} \quad \text { is stationary in the sense of }(5), \\
\mathbb{E}\left(\int_{\Phi(Q, \cdot)} \nabla w_{p}(y, \cdot) d y\right)=0 .
\end{array}\right.
$$

\subsection{Small random perturbations of the periodic setting}

The next step is to superimpose to the setting defined in the previous section an actual assumption that encodes that the material considered is a small perturbation of a periodic material. This is achieved by writing (see Figure 1)

$$
\Phi(x, \omega)=x+\eta \Psi(x, \omega)+O\left(\eta^{2}\right) .
$$

It has been shown in [10] that, when $\Phi$ in (8) is such a perturbation of the identity map, the solution to the corrector problem (13) may be developed in powers of the small parameter $\eta$. It reads $\widetilde{w}_{p}(x, \omega)=$ 


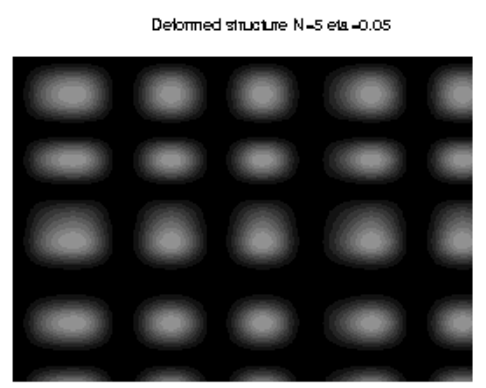

FigURE 1. We show a particular realization of $A_{p e r} \circ \Phi^{-1}(x, \omega)$ for $\eta=0.05$ in (14). Reprinted from [17].

$w_{p}^{0}(x)+\eta w_{p}^{1}(x, \omega)+O\left(\eta^{2}\right)$, where $w_{p}^{0}$ solves

$$
-\operatorname{div}\left[A_{p e r}\left(p+\nabla w_{p}^{0}\right)\right]=0, \quad w_{p}^{0} \text { is } Q \text {-periodic, }
$$

and where $w_{p}^{1}$ solves

$$
\left\{\begin{array}{l}
-\operatorname{div}\left[A_{p e r} \nabla w_{p}^{1}\right]=\operatorname{div}\left[-A_{p e r} \nabla \Psi \nabla w_{p}^{0}-\left(\nabla \Psi^{T}-(\operatorname{div} \Psi) \operatorname{Id}\right) A_{p e r}\left(p+\nabla w_{p}^{0}\right)\right], \\
\nabla w_{p}^{1} \text { is stationary and } \mathbb{E}\left(\int_{Q} \nabla w_{p}^{1}\right)=0 .
\end{array}\right.
$$

The problem (16) in $w_{p}^{1}$ is random in nature, but it is in fact easy to see, taking the expectation, that $\bar{w}_{p}^{1}=\mathbb{E}\left(w_{p}^{1}\right)$ is $Q$-periodic and solves the deterministic problem

$$
-\operatorname{div}\left[A_{p e r} \nabla \bar{w}_{p}^{1}\right]=\operatorname{div}\left[-A_{p e r} \mathbb{E}(\nabla \Psi) \nabla w_{p}^{0}-\left(\mathbb{E}\left(\nabla \Psi^{T}\right)-\mathbb{E}(\operatorname{div} \Psi) \operatorname{Id}\right) A_{p e r}\left(p+\nabla w_{p}^{0}\right)\right] .
$$

This is useful because, on the other hand, the knowledge of $w_{p}^{0}$ and $\bar{w}_{p}^{1}$ suffices to obtain a first order expansion (in $\eta$ ) of the homogenized matrix. Define $A_{i j}^{0}=\int_{Q}\left(e_{i}+\nabla w_{e_{i}}^{0}\right)^{T} A_{p e r} e_{j}$ and

$$
A_{i j}^{1}=-\int_{Q} \mathbb{E}(\operatorname{div} \Psi) A_{i j}^{0}+\int_{Q}\left(e_{i}+\nabla w_{e_{i}}^{0}\right)^{T} A_{p e r} e_{j} \mathbb{E}(\operatorname{div} \Psi)+\int_{Q}\left(\nabla \bar{w}_{e_{i}}^{1}-\mathbb{E}(\nabla \Psi) \nabla w_{e_{i}}^{0}\right)^{T} A_{p e r} e_{j},
$$

we then have

$$
A_{*}=A^{0}+\eta A^{1}+O\left(\eta^{2}\right) .
$$

As subsequently shown in [17], a similar approach can be applied to the problem once discretized by a finite element approach.

\subsection{Rare but possibly large random perturbations}

One (among many other possible -see the references on this issue-) different perturbative approach, described in full details in [2-4], and again addressing the random material as a small perturbation of a periodic material, consists in considering

$$
A_{\eta}(x, \omega)=A_{p e r}(x)+b_{\eta}(x, \omega) C_{p e r}(x)
$$




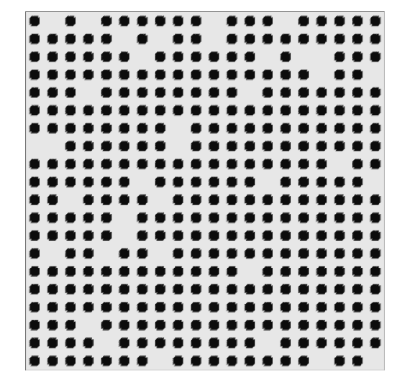

Figure 2. A typical random realization of the Bernoulli law for the perturbed periodic material.

where, with evident notation, $A_{p e r}$ is a periodic matrix modeling the unperturbed material, and $C_{p e r}$ is a periodic matrix modeling the perturbation. Consider then the case

$$
b_{\eta}(x, \omega)=\sum_{k \in \mathbb{Z}^{d}} \mathbf{1}_{\{Q+k\}}(x) B_{\eta}^{k}(\omega),
$$

where the $B_{\eta}^{k}$ are, say, independent identically distributed random variables. One particularly interesting case (see [2-4] for other cases) is when the common law of the $B_{\eta}^{k}$ is assumed to be a Bernoulli law of parameter $\eta$ (see Figure 2). Intuitively, it is difficult to expand in "powers" of $b_{\eta}$ because $B^{p}=B$ for all $p$ for a Bernoulli random variable. We now explain a formal approach, for which we do not know any rigorous foundation to date, except the recent work [28] which is a first step towards a mathematical grounding for our ideas. Although definite conclusions on the validity of the approach have yet to be obtained, the numerical tests we have performed (and reported upon in [3]) show its practical rectitude and efficiency.

The starting point of the formal argument consists in noticing that in the corrector problem

$$
-\operatorname{div}\left[A_{\eta}(y, \omega)\left(p+\nabla w_{p}(y, \omega)\right)\right]=0,
$$

the only source of randomness comes from the coefficient $A_{\eta}(y, \omega)$. Therefore, in theory, if one knows the law of this coefficient, one knows the law of the corrector function $w_{p}(y, \omega)$ and therefore may compute the homogenized coefficient $A^{*}$, the latter being a function of this law. When the law of $A_{\eta}$ is an expansion in terms of a small coefficient, so is the law of $w_{p}$. Consequently $A_{\eta}^{*}$ can be obtained as an expansion. Heuristically, on the cube $Q_{N}=[0, N]^{d}$ and at order 1 in $\eta$, the probability to get the perfect periodic material (entirely modeled by the matrix $\left.A_{\text {per }}\right)$ is $(1-\eta)^{N^{d}} \approx 1-N^{d} \eta+O\left(\eta^{2}\right)$, while the probability to obtain the unperturbed material on all cells except one (where the material has matrix $A_{p e r}+C_{p e r}$ ) is $N^{d}(1-\eta)^{N^{d}-1} \eta \approx N^{d} \eta+O\left(\eta^{2}\right)$. All other configurations, with more than two cells perturbed, yield contributions of orders higher than or equal to $\eta^{2}$. This gives the intuition (and this intuition can be turned into a mathematical proof when one restricts the problem to considering truncations of the corrector problem on large domains $Q_{N}$, see [3]) that the first order correction indeed comes from the difference between the material perfectly periodic except on one cell and the perfect material itself (see Figure 3 ). At least formally, $A_{\eta}^{*}=A_{p e r}^{*}+\eta A_{1, *}+o(\eta)$ where $A_{p e r}^{*}$ is the homogenized matrix for the unperturbed periodic material and

$$
A_{1, *} e_{i}=\lim _{N \rightarrow+\infty} \int_{Q_{N}}\left[\left(A_{p e r}+\mathbf{1}_{Q} C_{p e r}\right)\left(\nabla w_{i}^{N}+e_{i}\right)-A_{p e r}\left(\nabla w_{i}^{0}+e_{i}\right)\right],
$$

where $w_{i}^{0}$ is the corrector for $A_{\text {per }}$, and $w_{i}^{N}$ solves

$$
-\operatorname{div}\left(\left(A_{\text {per }}+\mathbf{1}_{Q} C_{\text {per }}\right)\left(\nabla w_{i}^{N}+e_{i}\right)\right)=0 \quad \text { in } \quad Q_{N}, \quad w_{i}^{N} Q_{N} \text { - periodic. }
$$


Note that the integral appearing in the right-hand side of (21) is not normalized: it a priori scales as the volume $N^{d}$ of $Q_{N}$ and has finite limit only because of cancellation effects between the two terms in the integrand.
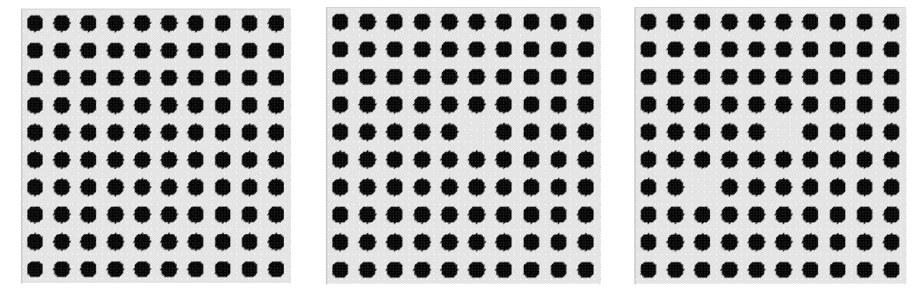

FiguRe 3. From left to right: zero defect, one defect and two defects.

The approach has been extensively tested. It is observed that, using the perturbative approach, the large $N$ limit for cubes of size $N$ is already very well approached for small values of $N$. As in the previous section, the computational efficiency of the approach is clear: solving the two periodic problems with coefficients $A_{\text {per }}$ and $A_{\text {per }}+\mathbf{1}_{Q} C_{\text {per }}$ for a limited size $N$ is much less expensive than solving the original, random corrector problem for a much larger size $N$.

An interesting follow-up is examined in [24]: when the second order term is needed, configurations with two defects have to be computed. They all can be seen as a family of PDEs, parameterized by the geometrical location of the defects (see Figure 3). Reduced basis techniques have been shown to be useful and allow for a definite speed-up in the computation.

\section{VARIANCE ISSUES IN STOCHASTIC HOMOGENIZATION}

Suppose now we have to solve the random problem (6), but that no satisfactory approximation can be performed using the techniques described above. The genuine random problem then needs to be addressed. Variance issues are now central to the practical approximation. We show two possible appropriate techniques that, for finite $N$, allow to compute the approximation $A_{N}^{\star}$ more effectively, that is, with a smaller variance. The present section summarizes arguments and computations more exhaustively developed in $[6,7,18]$ and in $[27]$.

\subsection{Antithetic variables}

We present here a first attempt to reduce the variance in stochastic homogenization. For this purpose, we consider a simple situation. The equation under consideration is the same simple elliptic equation in divergence form considered above, with a scalar coefficient. The coefficient is assumed to consist of independent, identically distributed random variables set on a simple mesh. The technique used for variance reduction is that of antithetic variables.

Setting. The direct approach for numerical random homogenization consists in using empirical means approximating the expectation $\mathbb{E}\left(A_{N}^{\star}\right)$ of the homogenized coefficient computed on a truncated domain of size $N$.

The variance reduction technique will consist in concurrently considering two sets of configurations for the random material instead of only one set. The two sets of configurations will be deduced one from the other. Indeed, fix $M=2 \mathcal{M}$. Suppose that we give ourselves $\mathcal{M}$ i.i.d. copies $\left(A^{\mathbf{m}}(x, \omega)\right)_{1 \leq \mathbf{m} \leq \mathcal{M}}$ of $A(x, \omega)$. Construct next $\mathcal{M}$ i.i.d. antithetic random fields

$$
B^{\mathbf{m}}(x, \omega)=T\left(A^{\mathbf{m}}(x, \omega)\right), \quad 1 \leq \mathbf{m} \leq \mathcal{M},
$$

from the $\left(A^{\mathbf{m}}(x, \omega)\right)_{1 \leq \mathbf{m} \leq \mathcal{M}}$. The map $T$ transforms the random field $A^{\mathbf{m}}$ into another, so-called antithetic, field $B^{\mathbf{m}}$. The transformation is performed in such a way that, for each $\mathbf{m}, B^{\mathbf{m}}$ has the same law as $A^{\mathbf{m}}$, namely the law of the matrix $A$. Somewhat vaguely stated, if $A$ was obtained in a coin tossing game (using a fair coin), 
then $B^{\mathbf{m}}$ would be head each time $A^{\mathbf{m}}$ is tail and vice versa. Then, for each $1 \leq \mathbf{m} \leq \mathcal{M}$, we solve two corrector problems. One is associated to the original $A^{\mathbf{m}}$, the other one is associated to the antithetic field $B^{\mathbf{m}}$. Using its solution $v_{p}^{N, \mathbf{m}}$, we define the antithetic homogenized matrix $B_{N}^{\star, \mathbf{m}}$, the elements of which read, for $1 \leq i, j \leq d$,

$$
\left[B_{N}^{\star, \mathbf{m}}\right]_{i j}(\omega)=\frac{1}{\left|Q_{N}\right|} \int_{Q_{N}} e_{i}^{T} B^{\mathbf{m}}(\cdot, \omega)\left(e_{j}+\nabla v_{e_{j}}^{N, \mathbf{m}}(\cdot, \omega)\right) .
$$

And we finally set, for any $1 \leq \mathbf{m} \leq \mathcal{M}$,

$$
\widetilde{A}_{N}^{\star, \mathbf{m}}(\omega):=\frac{1}{2}\left(A_{N}^{\star, \mathbf{m}}(\omega)+B_{N}^{\star, \mathbf{m}}(\omega)\right)
$$

Since $A^{\mathbf{m}}$ and $B^{\mathbf{m}}$ are identically distributed, so are $A_{N}^{\star, \mathbf{m}}$ and $B_{N}^{\star, \mathbf{m}}$. Thus, $\widetilde{A}_{N}^{\star, \mathbf{m}}$ is unbiased (that is, $\left.\mathbb{E}\left(\widetilde{A}_{N}^{\star, \mathbf{m}}\right)=\mathbb{E}\left(A_{N}^{\star, \mathbf{m}}\right)\right)$. In addition, it satisfies:

$$
\widetilde{A}_{N}^{\star, \mathbf{m}} \underset{N \rightarrow+\infty}{\longrightarrow} A^{\star} \text { almost surely }
$$

because $B^{\mathbf{m}}$ is ergodic. The hope is that the new approximation $\widetilde{A}_{N}^{\star, \mathbf{m}}$ has less variance than the original one $A_{N}^{\star, \mathbf{m}}$. It is indeed the case under appropriate assumptions.

Foundation of the approach. In $[6,7,18]$, we have provided theoretical elements that give a foundation to our approach of variance reduction. Of course, as always in this context, the one-dimensional setting, being explicit, may be entirely analyzed. We have demonstrated there the interest of the approach. Two higher dimensional cases were also analyzed. The first one is a "genuinely" random setting, the second one is a "weakly random" case, in the spirit of a case previously considered in Section 2. In both cases, under appropriate assumptions, the approach is shown to qualitatively reduce the variance. A quantitative assessment of the reduction is however out of reach. Only numerical tests can provide some information in this direction.

Numerical tests. The tests we have performed in $[6,18]$ concern three different "input" random fields $A(\cdot, \omega)$ in (6), some i.i.d., some correlated, with various correlation lengths. We equip these three settings with appropriate assumptions so that the elliptic problem (6) is well posed. In these settings, we have investigated variance reduction on a typical diagonal $\left[A_{N}^{\star}\right]_{11}$, or off-diagonal $\left[A_{N}^{\star}\right]_{12}$ entry of the approximate homogenized matrix $A_{N}^{\star}$, as well as on the eigenvalues of the matrix, and the eigenvalues of the associated differential operator $L_{A}=-\operatorname{div}\left[A_{N}^{\star}(\omega) \nabla \cdot\right]$ (supplied with homogeneous Dirichlet boundary conditions on $\left.\partial \mathcal{D}\right)$.

Our numerical results show that the technique may be applied to a large variety of situations and has proved efficient whatever the output considered. Variance is systematically reduced. We observed however that the rate of reduction is not spectacular. This has motivated the consideration of an alternative technique, expected to be, in several contexts, more efficient than antithetic variables.

\subsection{Control variate technique}

The control variate approach is a variance reduction technique known to be potentially much more efficient than the antithetic variable technique. It however asks to have beforehand a better information on the random variable simulated. In the context of homogenization, the work [27] presents a first possible investigation of the efficiency of this technique.

The specific setting considered is that of Section 2.3 above, namely a periodic setting perturbed using a random field modeled by a Bernoulli variable. Except that, now, the parameter $\eta$ of the Bernoulli law is not taken small. The expansion technique employed in Section 2.3 is therefore inaccurate. It can however serve for the construction of a control variate, useful to reduce the variance. 
In slightly more details, the technique for reducing the variance can be outlined as follows. We recall the field $b_{\eta}$ writes as in (19), that is $b_{\eta}(x, \omega)=\sum_{k \in \mathbb{Z}^{d}} \mathbf{1}_{\{Q+k\}}(x) B_{\eta}^{k}(\omega)$. Determining the field $A(x, \omega)$, given by $(18)$, on the truncated domain $Q_{N}$ amounts to drawing $B_{\eta}^{k}(\omega)$ in each cell $Q+k$ in $Q_{N}$. This allows to compute the associated (approximate) homogenized coefficient $A_{N}^{\star}(\omega)$ from the solution to the corrector problem (20) truncated on $Q_{N}$. In parallel to this task, we reconstruct from the specific realization of the set of $B_{\eta}^{k}(\omega)$ a field that is used as a control variate. More precisely, we set

$$
C_{N}^{\star}(\omega)=A_{N}^{\star}(\omega)-\rho\left(A_{\mathrm{per}}^{\star}+A_{1}^{\star, N}(\omega)-\mathbb{E}\left[A_{\mathrm{per}}^{\star}+A_{1}^{\star, N}(\omega)\right]\right)
$$

In this formula,

$$
A_{1}^{\star, N}(\omega)=\frac{1}{\left|Q_{N}\right|} \sum_{k+Q \subset Q_{N}} B_{\eta}^{k}(\omega) \mathcal{A}_{k}^{1 \mathrm{def}}
$$

where $\mathcal{A}_{k}^{1 \text { def }}$ is the deterministic coefficient corresponding to the case of one defect located at position $k$ (it is actually independent of $k$ ). The parameter $\rho$ is a deterministic parameter, a traditional ingredient of control variate techniques, which is optimized in terms of the estimated variances of the objects at play. It is crucial to note that the expectation of $A_{1}^{\star, N}(\omega)$ is analytically computable. Since by construction $\mathbb{E}\left(C_{N}^{\star}(\omega)\right)=\mathbb{E}\left(A_{N}^{\star}(\omega)\right)$, the technique then consists in approximating the former (thus the latter) by an empirical mean. The theoretical study and the numerical tests in [27] show that the variance of $C_{N}^{\star}(\omega)$ being smaller than that of $A_{N}^{\star}(\omega)$, the quality of the approximation is improved.

\section{NOVEL DETERMinistic APPROACHES FOR NONPERIODIC SETTINGS}

We proceed with our endeavour to model disordered structures but we now follow a different route to model deviations from the periodic setting. We do not resort to any random modeling. We proceed entirely deterministically. Our starting point is a theory developed in [8] which allows for defining the energy per particle of infinite systems of interacting particles, provided the locations of these particles satisfy rather general geometric assumptions. To some extent, these assumptions are generalizations of more classical assumptions such as the prototypical periodicity assumption. The reader can for instance imagine the simple case of a periodic lattice where a finite number of sites have been displaced. It is clear that the local failure of periodicity is no obstacle to the definition of an energy per particle. Much more general perturbations can be considered. This is also the case in the context of homogenization. Our construction of general sets of points can be adapted to this context and be useful there, on a very abstract level. In contrast to the mathematical endeavours that also aim at addressing homogenization for very general structures, our own line of thought is to reach generality but not at the price of giving up explicitness. We want computable expressions for all the objects we manipulate: averages, homogenized coefficients, corrector functions. For instance, in the case of point defects, our idea, in homogenization, is to zoom in at the vicinity of a defect and compare with the periodic situation.

\subsection{An abstract setting}

In [8], we have considered an infinite set of points in $\mathbb{R}^{d}$, denoted by $\left\{X_{i}\right\}_{i \in \mathbb{N}}$, and have established some geometric properties that allow us to define its average energy. More precisely, we have proved that the following properties allowed us to define the average energy of the infinite set of particles $\left\{X_{i}\right\}_{i \in \mathbb{N}}$ for a large class of models:

(H1) $\sup _{x \in \mathbb{R}^{d}} \#\left\{i \in \mathbb{N} /\left|x-X_{i}\right|<1\right\}<+\infty$
(H2) $\exists R>0$ such that $\inf _{x \in \mathbb{R}^{d}} \#\left\{i \in \mathbb{N} /\left|x-X_{i}\right|<R\right\}>0$ 
(H3) for any $n \in \mathbb{N}$, the following limit exists

$$
\lim _{\varepsilon \rightarrow 0} \mu^{n}\left(\frac{x}{\varepsilon}, h_{1}, \cdots, h_{n}\right)=\nu^{n}\left(h_{1}, \cdots, h_{n}\right),
$$

(and is independent of $x$ ), where

$$
\mu^{n}\left(y, h_{1}, \cdots, h_{n}\right)=\sum_{i_{0} \in \mathbb{Z}^{d}} \sum_{i_{1} \in \mathbb{Z}^{d}} \cdots \sum_{i_{n} \in \mathbb{Z}^{d}} \delta_{\left(X_{i_{0}}, X_{i_{0}}-X_{i_{1}}, \cdots, X_{i_{0}}-X_{i_{n}}\right)}\left(y, h_{1}, h_{2}, \cdots, h_{n}\right) .
$$

Intuitively, (H1) means there is no arbitrarily large cluster of particles, whereas (H2) means there is no arbitrarily large ball in $\mathbb{R}^{d}$ containing none of the $X_{i}$. The assumptions (H1)-(H2) are usually referred to as the "Delaunay" hypotheses. It is actually a variant of Assumption (H3) that has been originally introduced in [8] because, as briefly mentioned above, we were dealing there with energies of infinite interacting particle systems. The context of homogenization being related, but not identical, the assumptions are slightly different.

The relation between all these assumptions, and their relation with other conditions such as those of the random stationary ergodic context, or variants of the latter context such as those seen earlier in this article, is examined in details in [10]. form

We next introduce $\mathcal{A}^{k, p}\left(\left\{X_{i}\right\}\right)$, the closure for the $W_{\text {unif }}^{k, p}$ norm of the algebra generated by functions of the

$$
f(x)=\sum_{i \in \mathbb{N}} \varphi\left(x-X_{i}\right), \quad \varphi \in \mathcal{D}\left(\mathbb{R}^{d}\right) .
$$

We of course point out that in the particular case of a periodic lattice $\left\{X_{i}\right\}_{i \in \mathbb{N}}, \mathcal{A}^{k, p}\left(\left\{X_{i}\right\}_{i \in \mathbb{N}}\right)$ consists of periodic functions with the appropriate period and regularity.

The point is, any function $f$ in the spaces $\mathcal{A}^{k, p}$ has an average (see the proof in [8]), that is, the following limit exists:

$$
\langle f\rangle=\lim _{R \rightarrow \infty} \frac{1}{\left|B_{R}\right|} \int_{B_{R}} f .
$$

Two simple nonperiodic examples of $\left\{X_{i}\right\}$ satisfying our assumptions are the case of a compactly perturbed periodic system, and the case of two semi-crystals glued together. Both cases, although simple, are extremely relevant practically.

We wish to again emphasize two differences between the endeavour we outline here and other existing works in the literature. The theory of $H$-homogenization allows to homogenize very general problems and completely settle the issues. However, it does not always provide explicit expressions for the objects manipulated: "the" corrector function may be only a sequence of functions, not necessarily accessible to computations, and the homogenized coefficients may be obtained only as weak limits. Designing a numerical strategy to approximate these coefficients is unclear. Likewise, the theory developed by G. Nguetseng in $[29,30]$ (and summarized in [10]) also allows, in a different direction, to obtain homogenized limits for very general settings. But the generality comes at the price of losing the explicitness of the objects (homogenized coefficients, correctors). And the numerical approximation of those objects is then unclear.

The purpose of our mathematical endeavour is to keep the setting as general as possible, but also ensure that there are formulae amenable to computational approximations for all the objects the homogenization procedure creates. Several issues in this direction are currently under investigation [12]. Many actual situations considered are related to the modeling of defects, but our work is still in too preliminary a stage to be considered in the present review. So we prefer to consider an actual concrete case where the types of ideas developed above already bring an actual added value. The study considers a local defect in a periodic structure and shows how the paradigm of classical homogenization can be slightly twisted to develop interesting theoretical remarks and a corresponding numerical approach. 


\subsection{An example of practical setting}

We now consider (equipped with the appropriate assumptions so that the problem is well-posed) the equation

$$
-\operatorname{div}\left(a(x / \varepsilon) \nabla u^{\varepsilon}\right)=f
$$

with

$$
a=a_{\text {per }}+b
$$

where $a_{\text {per }}$ is a periodic function and $b \in L^{2}\left(\mathbb{R}^{d}\right)$ models a localized perturbation $b$. We see this mathematical example as an attempt to model a material with a periodic microstructure in the presence of a localized defect. The specific case considered here makes essential use of the Hilbert structure of $L^{2}\left(\mathbb{R}^{d}\right)$. More general cases of perturbations (such as $b$ in functional spaces other than $L^{2}\left(\mathbb{R}^{d}\right)$ ) give rise to much more substantial mathematical difficulties and will be discussed in [12].

Our purpose is then to theoretically derive an approximation of the solution $u^{\varepsilon}$ to (24), which is less expensive to compute than $u^{\varepsilon}$ itself but everywhere accurate at the small scale $\varepsilon$. It is indeed easy to realize that periodic homogenization can be readily applied to (24)-(25). Considering the homogenized equation

$$
-\operatorname{div}\left(A^{*} \nabla u^{*}\right)=f
$$

(where $A^{*}$ is defined as in the periodic case and is actually independent of $b$ ) provides an approximation for $u^{\varepsilon}$ and, using the periodic corrector, its gradient $\nabla u^{\varepsilon}$. All this will be briefly recalled below. The point is however that this approximation fails to accurately approximate the solution at the scale $\varepsilon x$ close to the "defect", that is where the perturbation $b$ is large. This is of course not an unexpected shortcoming of homogenization theory, since such an approximation property at the small scale is not the purpose of homogenization theory, the aim of which is to approximate the solution at scale one. We however believe that the need may arise, in practice, to obtain effective inexpensive approximations of $u^{\varepsilon}$ and its derivatives, at the small scale and in the vicinity of the defect. To this end, our approach consists, at practically no substantial additional computational cost, in computing a corrector $w$ better adapted to the problem than the periodic corrector $w_{\text {per }}$. Even though both give the same homogenized matrix $A^{*}$ and allow for the same quality of approximation of $u^{\varepsilon}$ at scale one, only the former performs uniformly well at scale $\varepsilon$.

On a more abstract level, the questions we investigate here are connected to the issues examined above: constructions of some general algebras and functional spaces that can be useful for homogenization theory. The specific functional space considered here is the space $L_{p e r}^{2}+L^{2}\left(\mathbb{R}^{d}\right)$ of sums of $L^{2}$ periodic functions and $L^{2}$ functions.

Outline of the approach, and theoretical results. Our main idea is to consider the natural, specific corrector function associated to the (nonperiodic) problem, namely the solution to

$$
-\operatorname{div}\left(\left(a_{p e r}+b\right)(y)\left(p+\nabla w_{p}(y)\right)\right)=0
$$

in $\mathbb{R}^{d}$ and proves that this function uniquely exists under appropriate conditions, and does a better job than the unperturbed periodic corrector (solution to the same equation as above with $b \equiv 0$ ) when approximating the solution $u^{\varepsilon}$ at the microscale, near the defect. Note that, although the above equation is linear elliptic and looks simple, even proving the existence of the solution to (27) can be a challenging theoretical issue for some perturbations $b$, or some more general coefficients than $a_{p e r}+b$. See [12].

The consideration of the one-dimensional version of (24) clearly shows (this particular example is worked out in [11] but the reader will have no difficulty to reproduce the argument independently) that when one considers the specific corrector $w$ solution to $-\frac{d}{d y}\left(\left(a_{p e r}+b\right)(y)\left(1+\frac{d}{d y} w(y)\right)\right)=0$, instead of the periodic corrector 
solution to $-\frac{d}{d y}\left(a_{p e r}(y)\left(1+\frac{d}{d y} w(y)\right)\right)=0$, then the quality of the (two-scale, first order) approximation of the solution $u^{\varepsilon}$ is immediately improved near the defect and at the scale of the defect.

In dimensions higher than or equal to two, the proof is slightly more involved, but not difficult. It is presented in [11] and theoretically demonstrates the interest of the approach. Under appropriate conditions, the solution $u^{\varepsilon}$ to $(24)$ is well approximated in $H^{1}$ norm, both at scale one and at scale $\varepsilon$, by the first order expansion $u^{\varepsilon, 1}(x)=u^{*}(x)+\varepsilon \sum_{i=1}^{d} \partial_{x_{i}} u^{*}(x) w_{e_{i}}(x / \varepsilon)$ constructed using the specific corrector $w$ (solution to (27)), in the following sense:

$$
\left\{\begin{array}{l}
\left\|\nabla\left(u^{\varepsilon}-u^{\varepsilon, 1}\right)\right\|_{L^{2}(\Omega)} \longrightarrow 0 \\
\left\|\nabla\left(u^{\varepsilon}-u^{\varepsilon, 1}\right)(\varepsilon \cdot)\right\|_{L^{2}\left(B_{R}\right)} \longrightarrow 0
\end{array}\right.
$$

where $B_{R}$ of course denotes any arbitrary fixed ball of radius $R$ such that $\varepsilon B_{R} \subset \Omega$. The latter approximation property does not in general hold for the periodic first-order approximation

$u_{\text {per }}^{\varepsilon, 1}(x)=u^{*}(x)+\varepsilon \sum_{i=1}^{d} \partial_{x_{i}} u^{*}(x) w_{e_{i}, p e r}(x / \varepsilon)$ constructed using the periodic corrector $w_{p, p e r}$.

A numerical illustration. We have performed some two-dimensional numerical tests in [11] in order to demonstrate the practical interest of the approach. For a specific case of coefficients $a_{\text {per }}$ and $b$, made precise there and encoding a periodic structure with an exponentially decaying defect around the origin, we have compared the quality of approximation at the vicinity of the "defect", that is, at the vicinity of the origin, obtained at scale $\varepsilon x$ using the first order expansion and, on the one hand the periodic corrector or, on the other hand, the actual corrector constructed above. More precisely, we look at

$$
\delta_{p e r}^{\varepsilon}=\frac{\left\|\nabla u^{\varepsilon}(\varepsilon \cdot)-\nabla u_{p e r}^{\varepsilon, 1}(\varepsilon \cdot)\right\|_{L^{2}(\Omega)}}{\left\|\nabla u^{\varepsilon}(\varepsilon \cdot)\right\|_{L^{2}(\Omega)}}, \delta^{\varepsilon}=\frac{\left\|\nabla u^{\varepsilon}(\varepsilon \cdot)-\nabla u^{\varepsilon, 1}(\varepsilon \cdot)\right\|_{L^{2}(\Omega)}}{\left\|\nabla u^{\varepsilon}(\varepsilon \cdot)\right\|_{L^{2}(\Omega)}} .
$$

Figure 4 and Table 1 present our results (all computations have been performed using a $P_{1}$ finite element approximation on triangular meshes - the meshsize $h$ is chosen sufficiently fine so that it does not perturb the results at the scale $\varepsilon^{-}$, and implemented using the software FreeFem $\left.++[20]\right)$. They unambiguously show the quality of the approximation provided by the non-periodic corrector, as opposed to the periodic one.
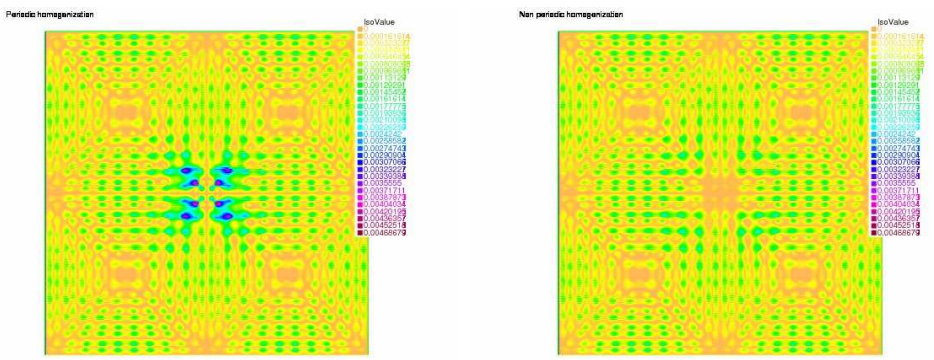

FiguRE 4. For $\varepsilon=1 / 10$, we display the norm $\left|\nabla u^{\varepsilon}(\varepsilon \cdot)-\nabla u_{\text {per }}^{\varepsilon, 1}(\varepsilon \cdot)\right|$ (left) and $\left|\nabla u^{\varepsilon}(\varepsilon \cdot)-\nabla u^{\varepsilon, 1}(\varepsilon \cdot)\right|$ (right). Recall that the defect is located at the origin, and has essentially size $\varepsilon$. The quality of approximation at the vicinity of the defect is significantly improved. Reproduced from [11]. 


\begin{tabular}{|c|c|c|}
\hline $1 / \varepsilon$ & $\delta_{\text {per }}^{\varepsilon}$ & $\delta^{\varepsilon}$ \\
\hline 3 & 0.198112 & 0.0850091 \\
\hline 5 & 0.191952 & 0.0425306 \\
\hline 10 & 0.183784 & 0.0266084 \\
\hline 20 & 0.175248 & 0.0139564 \\
\hline
\end{tabular}

TABLE 1. Numerical results: Relative errors (defined by (28)) using the periodic corrector (left column) and the corrector adapted to the case with defect (right column). Reproduced from [11].

\section{REFERENCES}

[1] A. Anantharaman, R. Costaouec, C. Le Bris, F. Legoll and F. Thomines, Introduction to numerical stochastic homogenization and the related computational challenges: some recent developments, in Lecture Notes Series, Institute for Mathematical Sciences, Vol. 22, eds. Weizhu Bao and Qiang Du (National University of Singapore, 2011), pp 197-272.

[2] A. Anantharaman and C. Le Bris, Homogénéisation d'un matériau périodique faiblement perturbé aléatoirement [Homogenization of a weakly randomly perturbed periodic material], C. R. Acad. Sci. Série I 348 (2010) 529-534.

[3] A. Anantharaman and C. Le Bris, A numerical approach related to defect-type theories for some weakly random problems in homogenization, SIAM Multiscale Modeling \& Simulation 9 (2011) 513-544.

[4] A. Anantharaman and C. Le Bris, Elements of mathematical foundations for a numerical approach for weakly random homogenization problems, Communications in Computational Physics 11 (2011) 1103-1143.

[5] A. Bensoussan, J. L. Lions, G. Papanicolaou, Asymptotic analysis for periodic structures, Studies in Mathematics and its Applications, Vol. 5 (North-Holland, 1978).

[6] X. Blanc, R. Costaouec, C. Le Bris and F. Legoll, Variance reduction in stochastic homogenization: the technique of antithetic variables, in Numerical Analysis of Multiscale Computations, B. Engquist, O. Runborg, R. Tsai, Eds., Lecture Notes in Computational Science and Engineering, Vol. 82, p 47-70, 2012.

[7] X. Blanc, R. Costaouec, C. Le Bris and F. Legoll, Variance reduction in stochastic homogenization using antithetic variables, Markov Processes and Related Fields, 18 (2012), 31-66.

[8] X. Blanc, C. Le Bris, and P.-L. Lions, A definition of the ground state energy for systems composed of infinitely many particles, Comm. P.D.E 28 (2003) 439-475.

[9] X. Blanc, C. Le Bris, and P.-L. Lions, On the energy of some microscopic stochastic lattices, Arch. Rat. Mech. Anal. 184 (2007) 303-340.

[10] X. Blanc, C. Le Bris, and P.-L. Lions, Stochastic homogenization and random lattices, J. Math. Pures Appl. 88 (2007) $34-63$.

[11] X. Blanc, C. Le Bris, and P.-L. Lions, A possible homogenization approach for the numerical simulation of periodic microstructures with defects, Milan J. Math., 80 (2012) 351-367.

[12] X. Blanc, C. Le Bris, and P.-L. Lions, in preparation

[13] A. Bourgeat and A. Piatnitski, Estimates in probability of the residual between the random and the homogenized solutions of one-dimensional second-order operator, Asymptotic Analysis, 21 (1999), 303-315.

[14] A. Bourgeat and A. Piatnitski, Approximation of effective coefficients in stochastic homogenization, Ann I. H. Poincaré $P R, \mathbf{4 0}(2)$ (2004), 153-165.

[15] S. Brenner and R. Scott, The Mathematical Theory of Finite Element Methods, 3rd Edition, Springer, New-York, 2008.

[16] E. Cancès And C. Le Bris, Mathematical modeling of point defects in materials science, Math. Mod. Meth. Appl. Sc., 23(10) (2013), 1795-1859.

[17] R. Costaouec, C. Le Bris and F. Legoll, Approximation numérique d'une classe de problèmes en homogénéisation stochastique, [Numerical approximation of a class of problems in stochastic homogenization], C. R. Acad. Sci. Série I 348 (2010), 99-103.

[18] R. Costaouec, C. Le Bris and F. Legoll, Variance reduction in stochastic homogenization: proof of concept, using antithetic variables, Bol. Soc. Esp. Mat. Apl., 50 (2010), 9-27.

[19] A. Gloria And F. Отто, An optimal variance estimate in stochastic homogenization of discrete elliptic equations, Ann. Prob., 39(3) (2011), 779-856. See also subsequent works by the same authors.

[20] F. Hecht, O. Pironneau, A. Le Hyaric, K. Ohtsuke, FreeFem++ (manual) http://www.freefem.org, 2007.

[21] V. V. Jikov, S. M. Kozlov and O. A. Oleinik, Homogenization of differential operators and integral functionals (SpringerVerlag, 1994).

[22] C. Le Bris, F. Legoll and F. Thomines, Multiscale Finite element approach for weakly random problems and related issues, M2AN, 48 (2014), 815-858. 
[23] C. Le Bris, Some numerical approaches for "weakly" random homogenization, Springer Lecture Notes in Computational Science and Engineering.G. Kreiss et al. (eds.), Numerical Mathematics and Advanced Applications 2009, p 29-45.

[24] C. Le Bris and F. Thomines, A Reduced Basis approach for some weakly stochastic multiscale problems, Chinese Ann. of Math. B, 33 (2012), 657-672.

[25] C. Le Bris, F. Legoll and A. Lozinski, MsFEM à la Crouzeix-Raviart for highly oscillatory elliptic problems, Chinese Ann. of Math. B, 34(1) (2013), 113-138.

[26] C. Le Bris, F. Legoll and A. Lozinski, MsFEM type approaches for perforated domains, Multiscale Model. Simul. 12(3) (2014), 1046-1077.

[27] F. Legoll And W. Minvielle, A control variate approach based on a defect-type theory for variance reduction in stochastic homogenization, arxiv.org/abs/1407.8029, preprint.

[28] J.-C. Mourrat, First order expansion of homogenized coefficients under Bernoulli perturbations, arxiv.org/abs/1301.7685, preprint.

[29] G. Nguetseng, Homogenization structures and applications I, Z. Anal. Anwendungen 22 (2003) 73-107.

[30] G. Nguetseng, Homogenization Structures and Applications II, Zeitschrift für Analysis und ihre Anwendungen 23 (2004) 483-508.

[31] A. Quarteroni And A. Valli, Numerical Approximation of Partial Differential Equations, Springer, Berlin, 1994. 